\title{
Investigation of sinus vein thrombosis cases detected in the emergency department
}

\author{
๑Ahmet Burak Erdem, @Elif Çelikel, @Muhittin Serkan Yılmaz, @Berkin Karapınarlı, @Uğur Bilgay Kaya \\ Ministry of Health Ankara City Hospital, Department of Emergency Medicine, Ankara, Turkey
}

Cite this article as: Erdem AB, Çelikel E, Yılmaz MS, Karapınarlı B, Kaya UB. Investigation of sinus vein thrombosis cases detected in the emergency department. J Health Sci Med 2021; 4(4): 428-433.

\begin{abstract}
Objective: Sinus vein thrombosis often presents to the emergency department with headache and is a rare disease among all strokes. Epidemiological data on sinus vein thrombosis are insufficient. Our aim in this study is to examine the findings of patients with sinus vein thrombosis who applied to our emergency department.

Material and Method: 267 patients with suspected sinus vein thrombosis in patients who came to our emergency department between 2013-2018 were included in the study. Patients were divided into 2 groups as present and without sinus vein thrombosis. Women were divided into 3 subgroups as not pregnant, pregnant and postpartum period. Demographic data, comorbid diseases, hemogram parameters and radiological data of the patients were recorded in a form. The relationship of the data with sinus vein thrombosis was examined.

Results: Sinus vein thrombosis was detected in $19.48 \%$ of the patients. $76.3 \%$ of the patients were women. $65.4 \%$ of them were between the ages of $18-40$. The most common complaint was headache with $51.9 \%$. And most of the patients' neurological examinations were normal. We found that hemoglobin $<8.75 \mathrm{~g} / \mathrm{dL}$ increased mortality and mean platelet volume was more effective in predicting mortality ( $\mathrm{AUC}=0.846$ ).

Conclusion: Sinus vein thrombosis is more common in women under 40 years of age. While headache is the most common reason for presentation, neurological examination is mostly normal. For this, magnetic resonance imaging and venography should be taken when the disease is suspected. Anemia and mean platelet volume may indicate poor prognosis.
\end{abstract}

Keywords: Anemia, female, headache, mean platelet volume (MPV), sinus vein thrombosis

\section{INTRODUCTION}

Venous vessels of the brain are divided into superficial and deep. The cortical veins and the superior sagittal sinus form the superficial veins. Deep veins, on the other hand, consist of transverse sinus, vein of Galen, straight and occipital sinuses and drain into dural sinuses. Finally, this structure drains into the vena jugularis interna (1). Thrombus in this venous circulation in the brain causes sinus vein thrombosis (SVT) (2). It is a rare type of stroke that is less common than arterial ischemic stroke and prevalent in women and young people $(2,3)$. Its frequency is estimated to be 1-12 per million per year (4). A study was conducted in the Netherlands demonstrating that its rate is $1.32-2.78$ per hundred thousand per year in women aged 31-50 years (5). While thrombophilia is the most important risk factor, pregnancy, postpartum period, oral contraceptive drugs, trauma, infection, cancer, and inflammatory diseases increase the risk of SVT (6). Although the symptoms of the patients are in a wide range, the most common type of it which we meet are headaches. Besides, nausea, blurred vision, loss of muscle strength, seizures, altered state of consciousness, and coma may be seen in patients. Neurological findings such as papilledema, cranial nerve findings, and paralysis can be detected (7). Magnetic resonance imaging (MRI) and MRI Venography (MRV) are used for diagnosis (8).

Although SVT is easier to be diagnosed after the occurrence of neurological symptoms, it might be diagnosed late when presented only with a headache. In this study, we aimed to reveal the characteristics of SVT patients diagnosed in the emergency service. 


\section{MATERIAL AND METHOD}

Our study was conducted with Ankara Numune Training and Research Hospital Ethics Committee (Date: 28/03/2019, Decision No: E-19-2627). Our study was conducted in accordance with the declaration of Helsinki and good clinical practices. There is no conflict of interest between the authors. Our study was planned as retrospectively observational. As it was in the form of file review over the hospital automation system and as it did not contain images that would enable the identification of the patients, the patients' consents were not obtained.

The files of 657 patients, who came to our emergency department between 2013 and 2018 and underwent magnetic resonance imaging (MRI) were examined. 267 patients over the age of 18 and having MRI venography were included in the study. 390 patients, who were excluded from the study, did not have MRI venography imaging. The imaging of different anatomical regions was done in these patients due to trauma and other reasons. A form was prepared for the study. The demographic data of the patients, their accompanying diseases, their complaints of presentation to the emergency service, neurological examination findings of them, pregnancy periods of women (divided into 3 groups as non-pregnant, pregnant, and postpartum period) were included in the study. In the relationship between SVT and age; they were grouped as follows: group 1: 18-40 years old, group 2: 41-65 years old, and group 3: 66 years old. Hemogram parameters were analyzed from laboratory values. Among these, Leukocyte (WBC-reference range (RA): 4.8-10.8 10\%/ $\mu \mathrm{L}$ ), Neutrophil (Neu-RA: 1.8-7.7 103/ $\mu \mathrm{L}$ ), Lymphocyte (Lym-RA: 1-4.8 $10^{3} / \mu \mathrm{L}$ ), Hemogram (Hgb-RA: 12-16 g/dL), Erythrocyte Distribution Width (RDW-RA: 11.5-14.5\% CV), Platelet (PLT-RA: 130-400 103/ $\mu \mathrm{L}$ ), Mean Platelet Volume (MPVRA: 7-12 fL), Platelet Distribution Width (PDW-RA: 9-17\%) were recorded. Computerized Brain Tomography (CBT) and MRI-MRV reports were examined from the radiological images of the patients. SVT status and other diseases detected in the patients were determined according to these reports and epicrisis records. In-hospital mortality of the patients was recorded. The data was recorded by two emergency medicine specialists. The other two emergency medicine specialists checked the data.

The patients were divided into two groups as with SVT and without SVT. Besides, three subgroups were created according to the pregnancy status of women as follows: no pregnancy, pregnant and postpartum period. Relationships between admission symptoms, neurological examination findings, hemogram parameters, CBT, and MRV results were examined among the groups. The presence of SVT according to the gestational period, which constitutes our subgroup, and the effects of the anatomical variation in the sinuses on the symptoms were statistically determined.
MRV is mostly used to confirm sinus vein thrombosis. In addition, observing isointense or hyperintense filling in the sinus in T1, T2, and FLAIR sequences is an important finding to distinguish SVT from anatomical variations (1). The postpartum period includes the 6-month period after the termination of pregnancy (9).

Venous blood samples were obtained in Vacutainer tubes of Becton Dickinson (BD diagnostics, Plymouth, UK) compatible with potassium EDTA. Samples were analyzed on XT-2000i (Sysmex Corporation of America, Long Grove, Illinois, USA). Computerized tomography system was Toshiba Aquilion 64-slice CT (Toshiba Medical System Corporation, Shimoishigami, Otawara-Shi, Japan). The magnetic resonance imaging device was 1.5 tesla (General Electric (GE) Healthcare, USA).

\section{Statistical Analysis}

SPSS 22.0 for Windows software was used for statistical analysis. Shapiro Wilks test was used to determine whether the data showed normal distribution. Descriptive statistical analyses were used to evaluate demographic data and data collected from tests and scales. Independent sample T-test was used to compare the data. Data were expressed as mean \pm standard deviation and percentages. $\mathrm{P}<0.05$ was considered statistically significant. In the independent sample T-test $\mathrm{p}$ values showed a statistically significant or non-significant inverse interaction.

\section{RESULTS}

Sinus vein thrombosis was detected in $19.48 \%$ of the patients. When we look at the gender and age distribution of SVT, $76.3 \%$ of those, who had SVT, were women. While the mean age of patients with SVT was 37.9, it was 34.7 for patients without SVT. $65.4 \%$ of those, who had SVT, were between the ages of $18-40$, and $11.5 \%$ of them were over 65 years old. No statistically significant difference was found between age groups $(p=0.152)$. The gender distribution and comorbid diseases of the patients are summarized in Table $\mathbf{1}$.

No significant difference was found between the patients' probability of having comorbid diseases $(p=0.581)$. The relationship of age and gender with SVT is summarized in Table 2.

When the relationship between SVT and general symptoms was examined by using an independent sample T-test, no difference was found between the two groups with $\mathrm{p}=0.435$. When the relationship between SVT and neurological examination findings was examined with an independent sample T-test, there was a significant relationship between the two groups with $\mathrm{p}=0.044$. It was seen that normal examination findings were higher with a rate of $75 \%(n=39)$ in those with SVT. These findings are summarized in Table 3. 


\begin{tabular}{|c|c|c|c|c|c|c|}
\hline \multirow{2}{*}{\multicolumn{2}{|c|}{ Variables }} & \multirow[b]{2}{*}{ SVT $^{*}$} & \multicolumn{2}{|c|}{$\mathbf{n}$} & \multicolumn{2}{|c|}{$\%$} \\
\hline & & & Yes & No & Yes & No \\
\hline \multirow{4}{*}{$\begin{array}{l}\overrightarrow{\tilde{U}} \\
\overrightarrow{0} \\
\tilde{U}\end{array}$} & \multicolumn{2}{|l|}{ Male } & 9 & 58 & 13.4 & 86.6 \\
\hline & \multirow{4}{*}{ Female } & Not pregnant & 22 & 100 & 18 & 82 \\
\hline & & Pregnant & 15 & 45 & 25 & 75 \\
\hline & & Postpartum & 6 & 12 & 33.3 & 66.7 \\
\hline \multirow{3}{*}{\multicolumn{3}{|c|}{$\begin{array}{l}\text { None } \\
\text { Diabetes mellitus }\end{array}$}} & \multicolumn{2}{|c|}{ Yes } & \multicolumn{2}{|c|}{ No } \\
\hline & & & \multicolumn{2}{|c|}{$39(75 \%)$} & \multicolumn{2}{|c|}{$162(75.3 \%)$} \\
\hline & & & \multicolumn{2}{|c|}{$3(5.8 \%)$} & \multicolumn{2}{|c|}{$7(3.3 \%)$} \\
\hline & \multicolumn{2}{|c|}{ Rheumatic disease } & \multicolumn{2}{|c|}{ - } & \multicolumn{2}{|c|}{$6(2.8 \%)$} \\
\hline & \multicolumn{2}{|l|}{ Eplepsy } & \multicolumn{2}{|c|}{$2(3.8 \%)$} & \multicolumn{2}{|c|}{$5(2.3 \%)$} \\
\hline & \multicolumn{2}{|c|}{ Preeclampsia } & \multicolumn{2}{|c|}{$1(1.9 \%)$} & \multicolumn{2}{|c|}{-} \\
\hline & \multicolumn{2}{|c|}{ Chronic renal failure } & \multicolumn{2}{|c|}{-} & \multicolumn{2}{|c|}{$1(0.5 \%)$} \\
\hline & \multicolumn{2}{|c|}{ Abortion } & \multicolumn{2}{|c|}{$1(1.9 \%)$} & \multicolumn{2}{|c|}{$2(0.9 \%)$} \\
\hline & Thyroid & sease & $2(3$ & & & \\
\hline$\ddot{z}$ & Sinus ve & thrombosis & $1(1$ & $\% \%)$ & & $3 \%)$ \\
\hline$\stackrel{\mathscr{\mathscr { D }}}{=}$ & Migrain & & & & 6( & 3\%) \\
\hline $\bar{Z}$ & Periana & scess & & & 4( & $\%)$ \\
\hline 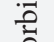 & Hyperte & & & & 1( & $5 \%)$ \\
\hline ఏ & Pseudo & nor cerebri & $2(3$ & 3\%) & 7( & $3 \%)$ \\
\hline$\overline{0}$ & Psychia & illness & & & & $5 \%)$ \\
\hline & Multipl & lerosis & & & & $5 \%)$ \\
\hline & Corona & artery disease & & & 2( & $\%)$ \\
\hline & Lympho & & & & & $5 \%)$ \\
\hline & Cerebro & scular disease & & & & $5 \%)$ \\
\hline & Deep ve & thrombosis & & & & $5 \%)$ \\
\hline & Periphe & vertigo & & & & $5 \%)$ \\
\hline & Trigemi & neuralgia & & & & $5 \%)$ \\
\hline & $\mathrm{HT}$ and & $S^{* *}$ & $1(1$ & $\%)$ & & \\
\hline
\end{tabular}

\begin{tabular}{|c|c|c|c|c|c|}
\hline \multicolumn{3}{|c|}{ Variables } & \multirow{2}{*}{$\frac{\text { SVT }^{*} \text { Yes }(\mathbf{n})}{9}$} & \multirow{2}{*}{$\begin{array}{c}\text { SVT No (n) } \\
58\end{array}$} & \multirow[t]{2}{*}{$\mathbf{p}$} \\
\hline \multirow{4}{*}{ Gender } & Male & & & & \\
\hline & \multirow{3}{*}{ Female } & Not pregnant & 22 & 109 & \\
\hline & & Pregnant & 15 & 50 & 0.149 \\
\hline & & Postpartum & 6 & 15 & \\
\hline \multirow{3}{*}{\multicolumn{2}{|c|}{ Age }} & $18-40$ & 34 & 156 & \\
\hline & & $41-65$ & 13 & 49 & 0.152 \\
\hline & & Above 66 & 5 & 10 & \\
\hline
\end{tabular}

Table 3. Relationship between symptoms and examination with MRV in patients with SVT

\begin{tabular}{|c|c|c|c|c|c|}
\hline \multirow{2}{*}{ Variables } & \multicolumn{2}{|c|}{ SVT $^{\star}$ Yes } & \multicolumn{2}{|c|}{ SVT No } & \multirow[b]{2}{*}{$\mathbf{p}$} \\
\hline & n & $\%$ & $\mathbf{n}$ & $\%$ & \\
\hline Headache & 27 & 51.9 & 96 & 44.6 & \\
\hline Nausea, vomiting, vertigo & 4 & 7.7 & 17 & 7.9 & \\
\hline Fever & - & - & 1 & 0.5 & \\
\hline ธิ Seizure & 4 & 7.7 & 23 & 10.7 & \\
\hline हี Defect of vision & 6 & 11.5 & 25 & 11.6 & \\
\hline के Consciousness change & 3 & 5.8 & 18 & 8.4 & 0.435 \\
\hline Loss of strength \& hypoesthesia & 7 & 13.5 & 32 & 14.9 & \\
\hline Speech disorder & 1 & 1.9 & 3 & 1.4 & \\
\hline Normal & 39 & 75 & 188 & 87.5 & \\
\hline Ptosis & 1 & 1.9 & 1 & 0.5 & \\
\hline .ี Pleji & 4 & 7.7 & 9 & 4.2 & 0.044 \\
\hline I Homonymous hemianopsia & - & & 3 & 1.4 & \\
\hline 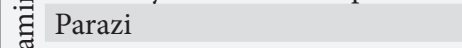 & 4 & 7.7 & 4 & 1.8 & \\
\hline Facial asymmetry & 1 & 1.9 & 5 & 2.3 & \\
\hline Cranial nerve sign & 3 & 5.8 & 4 & 1.8 & \\
\hline Aphasia & - & & 1 & 0.5 & \\
\hline
\end{tabular}

197 patients underwent CBT. Sixty-five of the 70 patients did not undergo CBT as they were pregnant, and five patients did not undergo CBT as they did not accept the tomography shooting. No acute pathology was found in the results of $172(87.4 \%)$ patients. $13(6.6 \%)$ patients with venous infarction in the CBT were detected and $12(6.1 \%)$ of them had SVT in MRV and 1 had sinus hypoplasia. Intracranial bleeding was seen in 4 (2\%) patients. SVT was detected in one of them. Intracranial mass was seen in $4(2 \%)$ patients, arterial ischemia in $2(1 \%)$, arteriovenous malformation in $1(0.5 \%)$ and cerebral edema in $1(0.5 \%)$ patient.

When the relationship between SVT and MRV is examined with an independent sample T-test; thrombus in MRV was detected as $100 \%(n=52)$ in patients with SVT. The correlation between those with general symptoms and MRV findings was investigated, but no relationship was established $(\mathrm{R}=0.025, \mathrm{p}=0,689)$. The correlation between those with neurological examination findings and MRV findings was investigated, but no relationship was established $(\mathrm{R}=-0.097, \mathrm{p}=0.127)$.

The mean values of the hemogram parameters are summarized in Table 4. When we look at whether there is a cut-off value between mortality and Hgb or not, the 'Area under the curve' (AUC) value was found to be 0.652 . However, an increase in mortality was observed if $\mathrm{Hgb}$ is $<8.75 \mathrm{~g} / \mathrm{dL}$ from the relationship between the cut-off value 1-specificity and sensitivity. Besides, the AUC value of MPV was found to be 0.846 . It was found to be more valuable in predicting mortality than other hemogram parameters. The AUC values of the hemogram parameters are shown in Figure 1.

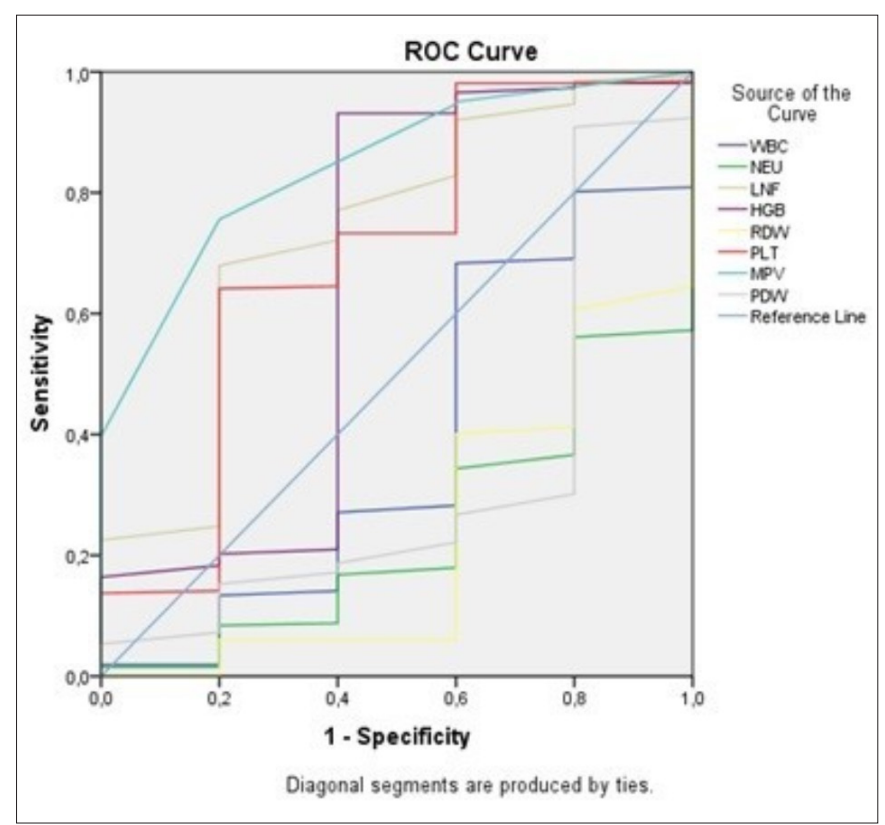

Figure 1. Relationship of hemogram parameters with mortality 
Table 4. Relationship between hemogram parameters and mortality

\begin{tabular}{|lcccc|}
\hline \multirow{2}{*}{ Variables } & \multicolumn{2}{c}{ SVT $^{*}$ Yes } & \multicolumn{2}{c|}{ SVT No } \\
\cline { 2 - 5 } Total & Alive (n) & Dead (n) & Alive (n) & \multicolumn{1}{c|}{ Dead (n) } \\
Leukocyte & 48 & 4 & 214 & 1 \\
Neutrophil & 10.4417 & 10.2500 & 10.0637 & 20.4000 \\
Lymphocyte & 7.4125 & 8.6500 & 6.8566 & 16.8000 \\
Hemoglobin & 2.1625 & 1.1500 & 2.3896 & 2.9000 \\
Red blood cell distribution width (RDW) & 13.2917 & 10.9000 & 13.2121 & 14.9000 \\
Thrombocyte & 14.2479 & 15.5500 & 14.1346 & 15.4 \\
Mean Platelet Volume (MPV) & 265.7917 & 180.2500 & 275.4121 & 346 \\
Platelet Distribution Width (PDW) & 9.2167 & 8.0000 & 9.2912 & 7.0 \\
\hline${ }^{*}$ SVT: Sinus vein thrombosis & 13.5271 & 15.1000 & 13.7703 & \\
\hline
\end{tabular}

\section{DISCUSSION}

In our study, we found that SVT is more common in women under 40 years of age in the emergency service and that the headache is the most common symptom. In these patients, we mostly could not find any findings on neurological examination. MRV was of high importance for diagnosis. We found that MPV, one of the hemogram values, was more significant than other parameters in predicting mortality.

Although the intercontinental rate of SVT varies, it is more common in women than in men (10). It is seen more than twice in women, with a rate of $74.5 \%$ (11). In our study, it was seen as $76.3 \%$ for women as in line with the literature. We believe that its higher prevalence in women is due to the fact that some of the disease's identified risk factors are only found in women. It is known that pregnancy, postpartum period, and use of oral contraceptives are important risk factors for SVT formation. This situation also causes the disease to be seen more frequently in young women (12). Our study contains results in this direction. 58.3\% of women diagnosed with SVT were in pregnancy or postpartum period. When we look at the age distribution in all patients, it was concentrated between the ages of 18-40 (65.4\%). While the mean age was 39.1 according to the International Study on Cerebral Vein and Dural Sinus Thrombosis (ISSVT) (11), it was found to be 41.24 according to the study by Sidhom (13) et al. In our study, we found it as 37.9. However, there was no statistical difference between age groups in the age distribution $(p=0.152)$. When we look at the ratio of SVT between genders in our study, the incidence of SVT was roughly 5 -times higher in women than in men. However, there was no statistical significance of SVT between genders ( $p>0.149$ ). This situation shows us that there are other risk factors besides gender and hormonal factors. Other risk factors include infection, coagulation disorder, malignancy, anemia, thrombocytosis, drugs, and trauma (14). As a limitation of our study, these data were missing from the records. However, 25\% $(n=13)$ of our patients with SVT had a comorbid disease. The most common accompanying disease was Diabetes mellitus (DM) $5.8 \%(n=3)$. It was found that $\mathrm{DM}$ was accompanying at the rate of $17 \%$ and $7 \%$ in SVT patients in some studies $(14,15)$.

Headaches are the most common cause of SVT. Besides, other symptoms are nausea, vomiting, visual impairment, focal neurological deficit, change in consciousness, epileptic seizure, speech disorder, and hypoesthesia $(2,10)$. While the headache was the most common reason (87.2\%) for admission in the VENOST study conducted by Duman et al. (2), Lee et al. (16) found this rate as $63.4 \%$. In our study, we found that headache (51.9\%) was the most common presenting symptom. In addition, hypoesthesia $13.5 \%$ and visual impairment $11.5 \%$ were other common symptoms. Despite the fact that the rate of headache is the most common symptom in research, the prevalence varies from each other. The reason for this may be due to the fact that SVT of all strokes is $<1 \%(10)$ and that the studies conducted did not include a great number of patients. Maali et al. (10) showed in their literature study that this rate varies between 28 $36 \%$ according to continents. Besides, the headache does not have a typical feature for SVT (7). In our study, we found that headache was statistically insignificant in determining SVT $(\mathrm{p}=0.435)$. This showed us that headache was an important symptom for SVT, but that it was insufficient for differential diagnosis. While focal neurological findings are $15 \%$ if the presentation is in good time, it can increase up to $50 \%$ when it is delayed (17). This rate was found to be $15.4 \%$ in our study. We think this is due to the early admission of patients to our emergency service and to the easy accessibility of our MRV facility. Kamışlı et al. (18) found the rate of neurological deficit to be $47 \%$. In our study, we found the presence of neurological findings to be statistically more significant in terms of SVT than patients without SVT $(\mathrm{p}=0.044)$. 
In emergency services, CBT is used for patients with neurological complaints. Its sensitivity is low in the diagnosis of SVT (19). In our study, 197 patients underwent CBT. Venous infarction was seen in 13 patients $(6.6 \%)$. The results of 12 patients $(6.1 \%)$ were consistent with MRV and SVT. Sinus hypoplasia was detected in 1 patient. MRI and MRV have sensitivity and specificity for the diagnosis of 95\% SVT (19). The European Stroke Organization (ESO) shows MRV as a good alternative to digital subtraction angiography (DSA) for the diagnosis of SVT (20). We showed thrombus along with MRV in all our patients whom we diagnosed with SVT. We detected SVT in 19.5\% $(n=52)$ of 267 patients suspected of SVT, and anatomical variation in the sinuses in $25.8 \%(n=69)$. The anatomical variation in the sinuses in MRV is between $25-36 \%$ and mostly it has no clinical result (21). When we looked at the correlation between the symptoms and findings of our patients and sinus variations in MRV, we could not find a correlation (respectively, $\mathrm{R}=0.025, \mathrm{p}=0.689$ / $\mathrm{R}=-0.097, \mathrm{p}=0.127$ ).

It has been shown that low Hgb may be a risk factor for SVT. Studies have reported that the association with anemia is between $7-27 \%$ in SVT patients (22). This situation has been attributed to the fact that anemia reduces the oxygen-carrying capacity and that it causes changes in blood viscosity (23). When we look at the mortality relationship between SVT and Hgb, we found AUC as 0.652. The fact that insufficient results in predicting mortality may be due to the low number of patients who died. Nevertheless, we analyzed a cutoff value between mortality and Hgb. The fact that Hgb decreases below $8.75 \mathrm{~g} / \mathrm{dL}$ showed that mortality increased. One study showed that SVT is associated with anemia at a rate of $30.67 \%$. As the anemia deepened, it was found that there was a poor outcome (endpoint) (23). Kamışlı et al. found a significant relationship between increased MPV value and SVT related to MPV, another one of the hemogram parameters. They concluded that this situation could be a predictor for poor prognostic outcomes (24). In our study, the AUC value of MPV was higher than the others $(\mathrm{AUC}=0.846)$. There are two different opinions about the relationship of MPV with thrombosis. It is thought MPV highness poses a risk for thrombus development. Increased volume of platelets may facilitate thrombus development. Another view is that the formed thrombus triggers the formation of a new platelet. Young and large platelets participate in the circulation. In addition, these young platelets respond poorly to antithrombotic therapies. These two theories are still debated. However, it is a common opinion that the increase of MPV is associated with poor outcome (25).

\section{Limitations}

The fact that it is a study conducted in a single-center hospital causes the number of patients to be low in terms of rare diseases. However, our easy access to MRV provided ease of obtaining the diagnosis in every patient we suspected. This enabled us to diagnose more than $19 \%$ of SVT among patients who undergone MRV. The retrospective nature of our study led to some data deficiencies. In particular, data on risk factors were insufficient. For us, the most important deficiencies in our study were 'the use of oral contraceptives, the absence of genetic research on coagulation disorder, and the lack of information on trauma and malignancy'. Our third limitation is that, due to our low mortality numbers, our analysis that we did with hemogram parameters caused insufficient results.

\section{CONCLUSION}

Especially newly developing headache that does not respond to analgesia in women under the age of 40 should suggest SVT. MRV shooting for diagnosis has a high confirmation rate. Hemogram is an analysis that is frequently checked in the emergency service. MPV is more significant in predicting mortality than other hemogram values. The retrospective nature of our study has some limitations. Therefore, multi-center prospective controlled studies will yield more specific epidemiological results for SVT. Besides, more studies are needed to reveal the relationship between $\mathrm{Hgb}$ and thrombocyte measurements and the prognosis of the disease. If anemia and increased MPV are indicators of poor prognosis, this situation may be a guide for early treatment of anemia and effective antithrombotic therapies for young thrombocytes.

\section{ETHICAL DECLARATIONS}

Ethics Committee Approval: The study was conducted with Ankara Numune Training and Research Hospital Ethics Committee (Date: 28/03/2019, Decision No: E-192627).

Informed Consent: Because the study was designed retrospectively, no written informed consent form was obtained from patients.

Referee Evaluation Process: Externally peer-reviewed.

Conflict of Interest Statement: The authors have no conflicts of interest to declare.

Financial Disclosure: The authors declared that this study has received no financial support.

Author Contributions: All of the authors declare that they have all participated in the design, execution, and analysis of the paper, and that they have approved the final version. 


\section{REFERENCES}

1. Kaya D. The Diagnosis and treatment of cerebral venous thrombosis. Turk J Neurol 2017; 23: 94-104.

2. Duman T, Uluduz D, Midi I, et al. A Multicenter study of 1144 patients with cerebral venous thrombosis: the VENOST study. J Stroke Cerebrovasc Dis 2017; 26: 1848-57.

3. Ferro JM, de Sousa DA. Cerebral venous thrombosis: an update. Curr Neurol Neurosc Rep 2019; 19: 1-9.

4. Ruiz-Sandoval JL, Chiquete E, Bañuelos-Becerra LJ, et al. Cerebral venous thrombosis in a Mexican multicenter registry of acute cerebrovascular disease: the RENAMEVASC study. J Stroke Cerebrovasc Dis 2012; 21: 395-400.

5. Coutinho JM, Zuurbier SM, Aramideh M, Stam J. The incidence of cerebral venous thrombosis: a cross-sectional study. Stroke 2012; 43: 3375-7.

6. Pate SI, Obeid H, Matti L, Ramakrishna H, Shamoun FE. Cerebral venous thrombosis current and newer anticoagulant treatment options. The Neurologist 2015; 20: 80-8.

7. Kısabay A, Eda Cakıroğlu, Deniz Selcuki. Cerebral venous thrombosis: a case series and literature review. Yoğun Bakım Derg 2015; 6: 48-56.

8. Masuhr F, Mehraein S, Einhaupl K. Cerebral venous and sinus thrombosis. J Neurol 2004; 251: 11-23.

9. Erçel Ö, Süt HK. Sleep quality and quality of life in postpartum woman. J Turk Sleep Med 2020; 1: 23-30.

10. Maali L, Sheema Khan S, Qeadan F, Ismail M, Ramaswamy D, Hedna VS. Cerebral venous thrombosis: continental disparities. Neurol Sci 2017; 38: 1963-8.

11. Ferro JM, Canhão P, Stam J, Bousser MG, Barinagarrementeria F. Prognosis of cerebral vein and dural sinus thrombosis. Stroke 2004; 35: 664-70.

12. Martinelli I, Passamonti SM, Maino A, et al. Pregnancy outcome after a first episode of cerebral vein thrombosis. J Thromb Haemost 2016; 14: 2386-93.

13. Sidhom Y, Mansour M, Messelmani M, et al. Cerebral venous thrombosis: clinical features, risk factors, and long-term outcome in a Tunisian cohort. J Stroke Cerebrovasc Dis 2014; 23: 1291-5.

14. Sassi SB, Touati N, Baccouche H, Drissi C, Romdhane NB, Hentati F. Cerebral venous thrombosis: a Tunisian monocenter study on 160 patients. Clin Appl Thromb Hemost 2017; 23: 1005-9.

15. English JD, Fields JD, Le S, Singh V. Clinical presentation and longterm outcome of cerebral venous thrombosis. Neurocrit Care 2009; 11:330-7.

16. Lee DJ, Ahmadpour A, Binyamin T, Dahlin BC, Shahlaie K, Waldau $B$. Management and outcome of spontaneous cerebral venous sinus thrombosis in a 5-year consecutive single-institution cohort. J Neuro Intervent Surg 2017; 9: 34-8.

17. Duran S, Özyurtlu D, Taşkapılığlu Ö, Hakyemez B, Bakar M. Cerebral venous sinus thrombosis: analysis of 27 cases. Turk J Cerebrovasc Dis 2014; 20: 87-93.

18. Kamışlı Ö, Arslan D, Altınayar S, Kamışlı S, Kablan Y, Özcan C. Sinus thrombosis: Clinical assessment. J Turk Cerebrovasc Dis $2009 ; 15 ; 39-42$.

19. Akgün H, Öz O, Taşdemir S, et al. Cerebral venous sinus thrombosis; analysis Of 17 cases. Gülhane Tip Derg 2014; 56: 102-6.

20. Ferro JM, Bousser MG, Canhao P, et al. European Stroke Organization guideline for the diagnosis and treatment of cerebral venous thrombosis - endorsed by the European Academy of Neurology. Eur J Neurol 2017; 24: 1203-13.

21. Freeman CW, Lazor JW, Loevner LA, Nabavizadeh SA. Variations of the CNS venous system mimicking pathology: spectrum of imaging findings. J Neuroimaging 2019; 29: 673-88.

22. Coutinho JM, Zuurbier SM, Gaartman AE, et al. Association between anemia and cerebral venous thrombosis: case-control study. Stroke 2015; 46: 2735-40.
23. Liu K, Song B, Gao Y, et al. Long-term outcomes in patients with anemia and cerebral venous thrombosis. Neurocrit Care 2018; 29 : 463-8.

24.Kamışlı Ö, Kamışlı S, Kablan Y,Gönüllü S, Özcan C. The prognostic value of an increased mean platelet volume and platelet distribution width in the early phase of cerebral venous sinus thrombosis. Clin App Thromb Hemost 2013; 19: 29-32.

25. Noris P, Melazzini F, Balduini CL. New roles for mean platelet volume measurement in the clinical practice? Platelets 2016; 27: 607-12. 